\title{
Amodal completion: Simplicity is not the explanation
}

\author{
LAURA MORAVEC and JACOB BECK \\ University of Oregon, Eugene, Oregon
}

\begin{abstract}
Six experiments were performed to investigate factors that might influence the use of symmetry versus good continuation in the completion of a partially occluded shape. With 1-sec presentations, subjects used good continuation on $74.6 \%$ of the trials and were not influenced by manipulations designed to draw their attention to different aspects of the shapes. With a 9-sec presentation, good continuation completions dropped to $37 \%$ and symmetric completions rose to $53.2 \%$. The results suggest a precedence effect with naive subjects. Features favoring completion through good continuation (e.g., lines at points of intersection) are read out of a sensory store more quickly than are features favoring completion through symmetry (e.g., symmetrically opposite corners). Showing only selected parts of the stimulus through holes in a mask influenced subjects' responses. The results suggest that completion is not based on the simplicity of the completed shape, but rather is determined by the parts of the stimulus that are processed.
\end{abstract}

Amodal completion refers to the perception of a shape that is not completely visible, as when one figure is partially covered by another. In Figure 1, for example, even though some contours are not visible, one senses that the contours are there and perceives a circle behind the square.

One important question about this phenomenon concerns how observers complete the partially hidden figures. Given the lack of actual perceived contours, there are an infinite number of possible completions. However, only a very small number of the possible completions are used. Most of the research has concentrated on the simplicity of the completed shape. Kanizsa (1975) presented evidence showing that although the Gestalt rules of simplicity and economy would lead us to expect symmetric completions whenever possible, observers more often used good continuation to complete partially hidden figures. Buffart, Leeuwenberg, and Restle (1981), using Leeuwenberg's coding theory, showed that in many instances, the good continuation and the symmetric completion are equally "simple," and therefore should occur equally often. However, in Kanizsa's demonstrations, and in many of the experiments discussed in this paper, good continuation was used significantly more often than symmetry to complete the figures, even when that process yielded a more asymmetric shape.

Six experiments were performed to investigate factors that might influence the use of symmetry versus good continuation in amodal completion. The first four experiments were manipulations of various stimulus factors in an attempt to bias the stimuli in favor of one of the two possible completions. The factors investigated included placement of a

This work is part of a master's thesis presented to the University of Oregon in 1979 by Laura Moravec. L. Moravec is currently at New College of the University of South Florida, 5700 N. Tamiami Trail, Sarasota, FL 34243-2197.

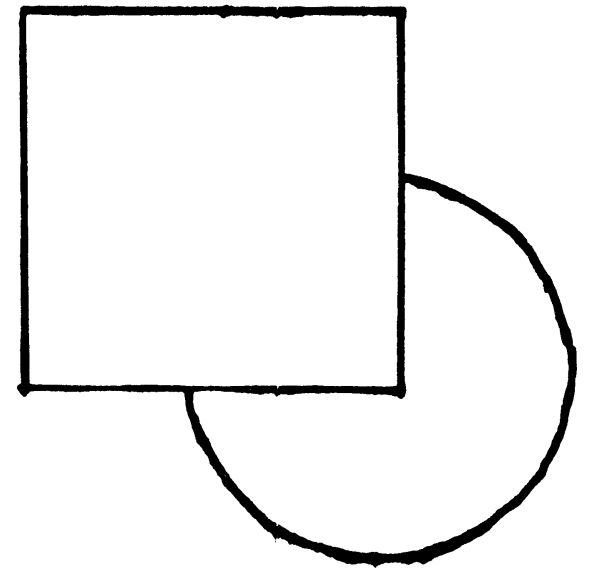

Figure 1. Example of an amodal completion stimulus. Observers reported perceiving a complete circle "behind" the square.

fixation point, brightness of the occluding or occluded shape, shape of the occluding figure, orientation of both shapes, peripheral versus foveal viewing, size, and presence of an example of the completion in the stimulus field.

All of the experiments were intended to manipulate the subjects' attention, to draw their attention to either the symmetric aspects or the good-continuation aspects of the stimulus. Discovering which biasing factor had the strongest influence on a subject's response would indicate which aspects of the figure subjects were processing.

\section{EXPERIMENTS 1-4}

\footnotetext{
Methods

Subjects. Undergraduates of the University of Oregon served as subjects in the experiments. There were 10 naive subjects in each of the four experiments (40 subjects total).

Stimuli. Figure 2 shows the three test stimuli used in all six of the experiments. Each of these shapes was partially occluded so that the
} 

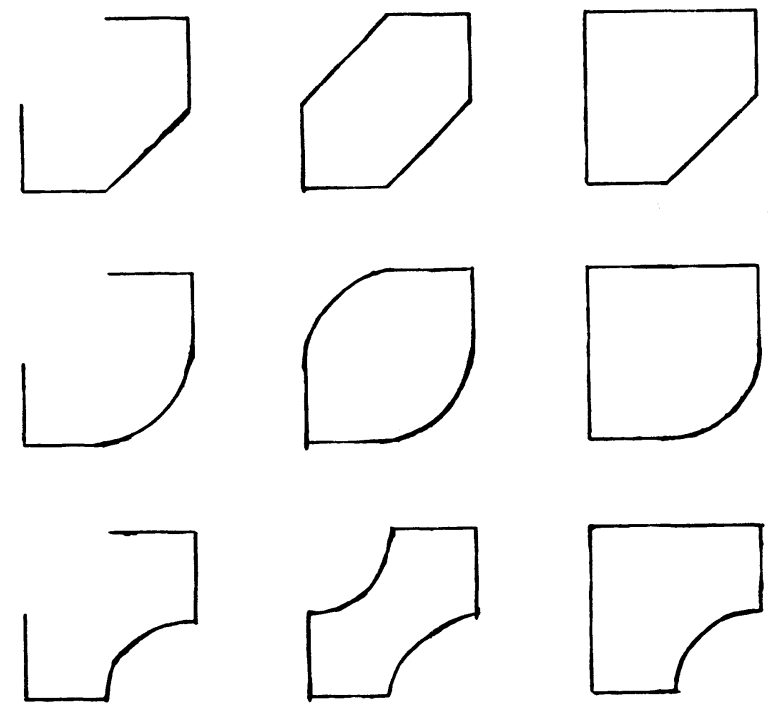

Figure 2. The three test stimuli used in all six experiments. The first column shows the sections visible during the experiments. The second column shows the symmetric completions and the third column shows the good-continuation completions.
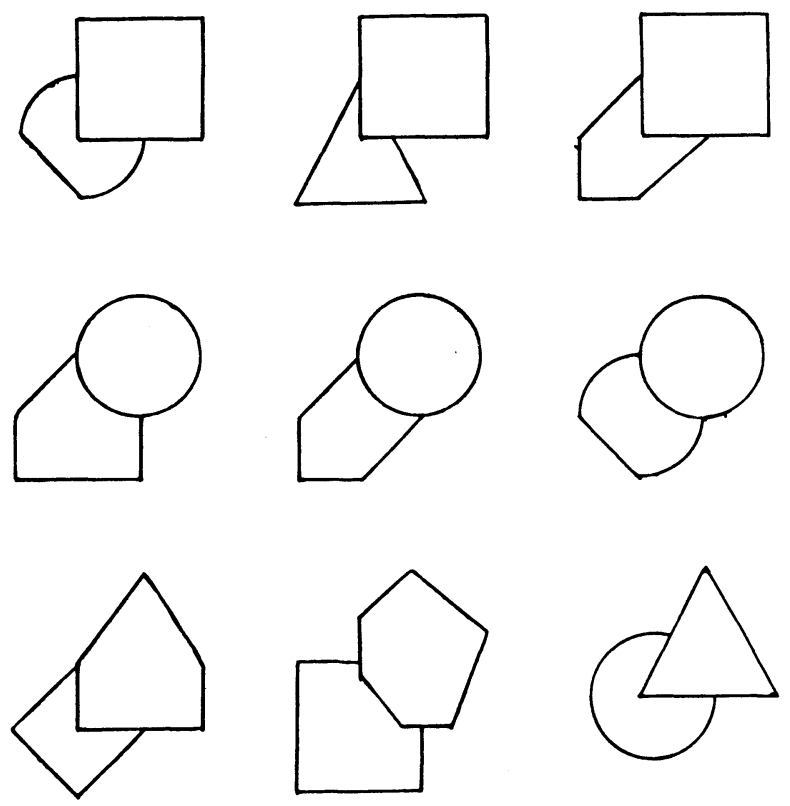

Figure 3. Examples of distractor stimuli used in the experiments. A variety of shapes were used for both the occluding and occluded figures. use of good continuation would lead subjects to add a corner and make an asymmetric shape. The use of the symmetry would lead subjects to add a straight line or a curve. Based on the appearance of the symmetric completion, these stimuli are referred to as the lozenge, the lemon, and the bow tie. Each experiment included a large number of distractor stimuli (on the average $\mathbf{4 0}$ per experiment). Figure 3 shows several examples of the distractor stimuli.

Pretests indicated no significant differences in subject responses based on whether the stimuli were line drawings (black ink on white paper) or were cut from colored construction paper.

Table 1 describes briefly the seven factors and how they were varied to produce the two different biases. Figure 4 shows an example of the two different biases for five of the seven factors investigated in the first four experiments.

Procedure. Pretests indicated no significant differences based on the mode of the subjects' responses. In some experiments, subjects responded verbally by saying whether the hidden part was a line, a corner, or a convex or concave curve. In other experiments, subjects drew the completions, and in others, subjects chose between three alternative completed shapes.

The stimuli were presented in a Gerbrands two-field tachistoscope for $1 \mathrm{sec}$ each.

\section{Results}

The proportion of symmetric, good-continuation, and other responses were entered into a chi-square test. None of the manipulations had a significant influence on the subjects' completion responses. Averaged over the four experiments, subjects used good continuation to complete the figure $74.6 \%$ of the time and used symmetry $19.4 \%$ of the time.

\section{EXPERIMENTS 5-6}

Experiment 5 used the same test and distractor stimuli. Subjects were required to look at each of three numbered fixation points for $3 \mathrm{sec}$ (9-sec presentation). Twenty naive subjects participated in this experiment. Although variations in the placement of the three fixation points had no influence, overall the average good-continuation completions dropped to $37 \%$, and symmetric completions rose to $53.2 \%$.

Good continuation is local factor in that only the two line segments at an intersection need to be processed for completion to occur. Symmetry is a more global factor in that more of the figure has to be processed to identify the symmetric completion. Symmetry, therefore, required a much greater processing of figural information than did good continuation. In a brief flash, good-continuation completions occur more frequently because the information for good continuation is read out first from the sensory store. With a more extended presentation, the stimu-

Table 1

Factors Used to Bias the Completion

\begin{tabular}{lll}
\hline Factor & Good-Continuation Bias & Symmetric Bias \\
\hline Fixation Point & Near corner of occluding shape & $\begin{array}{l}\text { Near part of shape symmetrically } \\
\text { opposite the hidden section } \\
\text { Occluded shape }\end{array}$ \\
Brightness & Occluding shape & Horizontal or vertical \\
Orientation & Diagonal & Symmetric completion \\
Example in Field & Good continuation completion & Symmetric completion \\
Shape of Occluding Figure & Square & 7 and 3.5 \\
Size & 14 of visual angle & Peripheral \\
Viewing & Foveal & \\
\hline
\end{tabular}



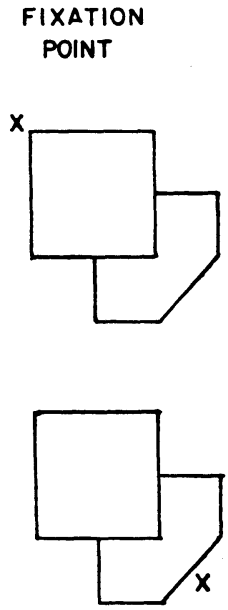

BRIGHTNESS
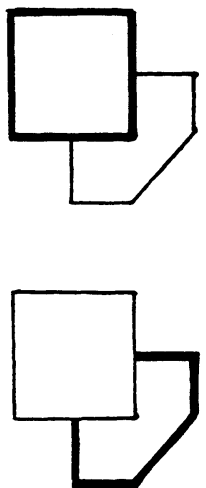

ORIENTATION
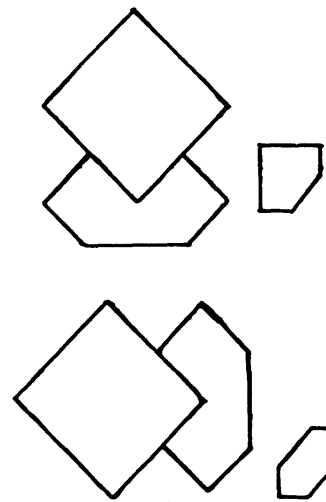

EXAMPLE IN FIELD
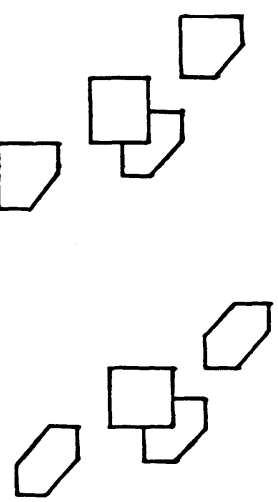

OCCLUDING SHAPE

Figure 4. Examples of two different biases for five of the seven factors investigated in the first four experiments. For all except the orientation manipulation, the top row provides examples biased for good continuation and the bottom row provides examples biased for symmetry. Both of the orientation examples are biased for symmetry in that a symmetric completion would involve a horizontal or vertical line.
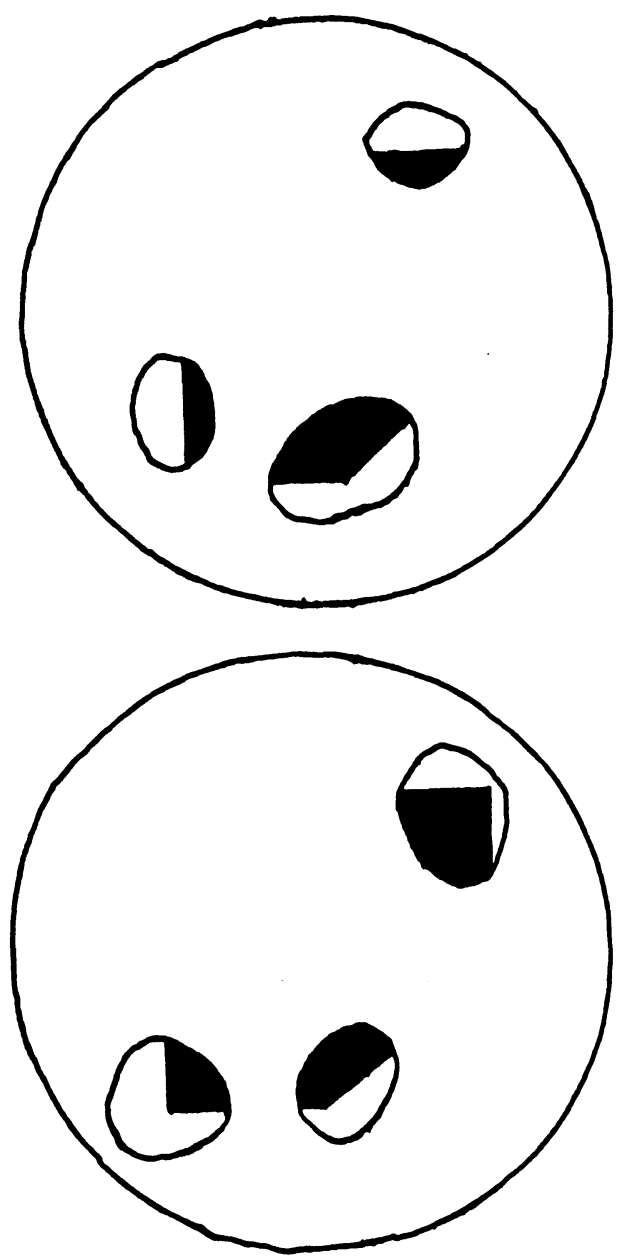

lus information for a symmetric completion is also encoded, increasing the number of symmetric completions.

In Experiment 6, a new type of stimulus was used. The lozenge, lemon, and bow tie were still the test stimuli. However, they were cut out of red construction paper and hidden behind a mask. The mask consisted of a circle (disk) cut out of black construction paper. Three to four holes were cut out of the disk in such a way that only specific parts of the occluded shape could be seen through the holes. Figure 5 shows two examples of masking the lozenge stimulus. One example is biased for symmetry by showing the symmetrically opposite corners and the area symmetrically opposite the section that is fully occluded. The other is biased for good continuation by assymmetric placement of the holes and showing a larger section of the area of the figure (the straight lines) leading toward the fully occluded section.

The displays were presented for $2 \mathrm{sec}$. The completion responses of 16 naive subjects were significantly influenced by the parts of the shape that were visible through the mask.

Averaged across subjects, the symmetrically biased stimuli were completed symmetrically $85 \%$ of the time. The good-continuation biased stimuli were completed with corners $67 \%$ of the time.

It is not known for certain why the biasing worked so well in the final experiment. It seems likely that specific features of the stimuli biased the subjects' responses. Whatever the reason, it is clear that subjects were not trying to produce the simplest possible shape.

\section{CONCLUSIONS}

Figure 5. Examples of the stimuli used in Experiment 6. The top example is biased for good continuation and the bottom for symmetry.

Amodal completion does not occur in the primary encoding of a pattern. Rather, the completion is determined 
by the specific parts of a stimulus that are processed when the subject is required to attend to the shape of the occluded figure. On trials with brief exposures $(1 \mathrm{sec})$, subjects appear to process only the interrupted lines that go "beneath" the occluding shape. With longer exposures $(3 \mathrm{sec})$, subjects have time to construct a number of possible completions. By manipulating the parts of the stimulus the subject sees, one can influence the construction process. In the first five experiments, all the information (the lines and their arrangement) was available to the subject. Attempts to draw the subjects' attention to specific parts of the drawing were not successful. Only when the subject was restricted to specific information did the manipulation have an influence.

\section{REFERENCES}

Buffart, H., Leeuwenberg, E., \& Restle, F. (1981). Coding theory of visual pattern completion. Journal of Experimental Psychology: Human Perception \& Performance, 7, 241-274.

KANIZSA, G. (1975). The role of regularity in perceptual organization. In G. Flores d'Arcais (Ed.), Studies in perception: Festschrift for Fabio Metelli. Milan: Martello-Giunti.

(Manuscript received for publication March 13, 1986.) 